\title{
Functional Homotopic Changes in Multiple Sclerosis with Resting-State Functional MR Imaging
}

\author{
Y. Zhou, M. Milham, X.-N. Zuo, C. Kelly, H. Jaggi, J. Herbert, R.I. Grossman, and Y. Ge
}

\begin{abstract}
BACKGROUND AND PURPOSE: CC is extensively involved in MS with interhemispheric dysfunction. The purpose of this study was to determine whether interhemispheric correlation is altered in MS by use of a recently developed RS-fMRI homotopy technique and whether these homotopic changes correlate with CC pathology.
\end{abstract}

MATERIALS AND METHODS: Twenty-four patients with relapsing-remitting MS and 24 age-matched healthy volunteers were studied with RS-fMRI and DTI acquired at 3T. The Pearson correlation of each pair of symmetric interhemispheric voxels of RS-fMRI time-series data was performed to compute VMHC, and z-transformed for subsequent group-level analysis. In addition, 5 CC segments in the midsagittal area and DTI-derived FA were measured to quantify interhemispheric microstructural changes and correlate with global and regional VMHC in MS.

RESULTS: Relative to control participants, patients with MS exhibited an abnormal homotopic pattern with decreased VMHC in the primary visual, somatosensory, and motor cortices and increased VMHC in several regions associated with sensory processing and motor control including the insula, thalamus, pallidum, and cerebellum. The global VMHC correlates moderately with the average FA of the entire CC for all participants in both groups $(r=0.3 ; P=.03)$.

CONCLUSIONS: Our data provide preliminary evidence of the potential usefulness of VMHC analyses for the detection of abnormalities of interhemispheric coordination in MS. We demonstrated that the whole-brain homotopic RS-fMRI pattern was altered in patients with MS, which was partially associated with the underlying structural degenerative changes of CC measured with FA.

ABBREVIATIONS: $C C=$ corpus callosum; $C S T=$ cortical spinal tract; $F A=$ fractional anisotropy; RSFC = resting-state functional connectivity; RS-fMRI $=$ restingstate functional MRI; $\mathrm{VMHC}=$ voxel-mirrored homotopic correlation

B rain homotopy describes a fundamental characteristic of the intrinsic functional architecture of the brain. Namely, it demonstrates that homologous regions in 2 hemispheres are geometrically and functionally corresponding to each other. Recently, such a high degree of interhemispheric synchrony can be estimated based on

Received June 6, 2012; accepted after revision August 17.

From the Radiology/Center for Biomedical Imaging (Y.Z., H.J., J.H., R.I.G., Y.G.) and New York University Child Study Center (M.M., X.-N.Z., C.K.), New York University School of Medicine, New York, New York; and Laboratory for Functional Connectome and Development (X.-N.Z.), Key Laboratory of Behavioral Science, Magnetic Resonance Imaging Research Center, Institute of Psychology, Chinese Academy of Sciences, Beijing, China.

Paper previously presented at: Annual Meeting of the American Society of Neuroradiology, June 4-9, 2011; Seattle, Washington.

This study was supported by the National Institutes of Health (grant R01NS029029) and the Natural Science Foundation of China (grant 81171409).

Please address correspondence to Yulin Ge, MD, Center for Biomedical Imaging, Department of Radiology, New York University School of Medicine, 660 First Ave, $4^{\text {th }}$ floor, New York, NY 10016; E-mail: yulin.ge@nyumc.org

- Indicates open access to non-subscribers at www.ajnr.org

http://dx.doi.org/10.3174/ajnr.A3386 spontaneous neuronal activity by use of RS-fMRI. ${ }^{1,2}$ By measuring bilateral regional RSFC, Stark et $\mathrm{al}^{1}$ showed a gradual distribution of interhemispheric functional correlation, with the highest found in primary sensorimotor cortices among ubiquitous brain homotopic regions and lower correlations found in the heteromodal association areas that are related to high-order functional lateralization. More recently, Zuo et $\mathrm{al}^{2}$ further extended the regional computation to a whole-brain voxel-based correlation map and showed marked and regionally specific age-related (ie, developmental and aging) changes in functional homotopy. Specifically, primary sensorimotor regions tended to show increasing homotopic RSFC, whereas higher-order processing regions showed decreasing interhemispheric connectivity with age. These studies provided robust evidence that brain homotopy can be reliably mapped with RS-fMRI-derived functional connectivity analysis. Although interhemispheric functional integration plays an important role in communicating perceptual, cognitive, learned, and volitional information, detailed clinical data on neurodegenerative and psychiatric disorders regarding brain functional homotopy are still very limited to date., 
MS is a white matter disease with diffuse neuronal degeneration. One of the remarkable histopathologic features of MS is its preferential and extensive involvement in the CC with demyelinating lesions often seen within callosal or subcallosal areas. ${ }^{5-7}$ The CC is the largest white-matter tract widely connecting bilateral neocortical areas and is the primary means of communication between the 2 hemispheres, ${ }^{8}$ making it a key structure in bilateral cortical-cortical functional activities. ${ }^{9}$ According to the known histologic pattern of the CC, through-CC cortical presentations (homotopic stimulations) yielded faster reaction times than heterotopic stimulations. ${ }^{10}$ Given extensive axonal degeneration and atrophy within the CC in MS, structural connectivity-related homotopy changes are likely. DTI has been particularly useful in determining the microstructural axonal degeneration in white matter tracts such as the CC by use of quantitative scalar indices such as mean diffusivity and FA. ${ }^{11}$ With use of fiber tractography obtained from DTI datasets, topographic distribution of fiber connections to the cortex through midsagittal cross-sections of the CC can be obtained by characterizing its directionality and connectivity in relationship to its cortical terminals. ${ }^{12}$ Using RSfMRI and DTI, several studies investigating the interhemispheric functional interactions of sensorimotor cortices in MS provided conflicting results: Lowe et $\mathrm{al}^{13}$ showed decreased functional connectivity, whereas Rocca et $\mathrm{al}^{14}$ found increased interhemispheric strengths of coefficients of effective connectivity. These studies, however, all focused on a specific sensoriomotor functional pathway; therefore, investigation of the changes of global transcallosal (ie, entire CC) structural and functional connectivity (ie, VMHC) is warranted to better understand the interhemispheric coordination deficits in MS.

We hypothesize that the decrements in the integrity of the CC in MS may affect the interhemispheric functional connecting properties or interactions (homotopy), which can be measured by VMHC, a recently developed voxelwise RSFC whole-brain analysis. ${ }^{2}$ Although the VMHC method is derived from RS-fMRI data, it provides unique information of functional homotopy between 2 hemispheres on the basis of voxelwise analysis; therefore, it is useful to detect interhemispheric coordination impairment in neurologic diseases (eg, MS).

The purpose of this study was to examine the interhemispheric synchronous changes of the brain by using RS-fMRI VMHC in patients with MS compared with healthy control participants. We also investigated the correlations of functional homotopic changes and microstructural changes of the CC by using DTI. In particular, we examined transcallosal and nontranscallosal cortical homotopic differences and changes, as well as their relationship with lesion load and Expanded Disability Status Scale score in patients with MS.

\section{MATERIALS AND METHODS \\ Patients}

Twenty-four patients with clinically definite relapsing-remitting MS (mean age, $38.25 \pm$ SD 9.23 years; 18 women and 6 men) and 24 age-matched healthy volunteers (mean age, $38.46 \pm$ SD 10.52 years; 10 women and 14 men) were recruited. None of the patients with MS had any evidence of or prior diagnosis of cardiovascular morbidity, cerebrovascular disease, or other neurologic diseases.
All patients displayed a characteristic phenotype that included but was not limited to visual disturbances (eg, diplopia), paresthesias, ataxia, dysphagia, fatigue, and nonspecific disabling arthralgias. The patients had a varied degree of previous symptoms with an average Expanded Disability Status Scale score of 2.47 (range, $0-6)$. The average disease duration for the patient group was 5.12 years (range, 0.67-14 years). The institutional review board approved this study. All participants gave informed consent after the nature of the procedure had been fully explained.

\section{MR Imaging}

RS-fMRI was performed on a 3T whole-body MR scanner (Magnetom Tim Trio; Siemens, Erlangen, Germany) by use of a 12channel head coil with a gradient-echo echo-planar imaging sequence (TR, $2 \mathrm{~s}$; TE, $30 \mathrm{~ms}$; flip angle, $75^{\circ}$; field of view, $220 \times 220$ $\mathrm{mm}^{2}$; and acquisition matrix size, $128 \times 128$ [153 volumes total]). A total of 20 sections were collected parallel to a line passing through the anterior/posterior commissure with $5-\mathrm{mm}$ section thickness and 1-mm gap and positioned to cover the entire cerebrum. In addition, sagittal magnetization-prepared rapid acquisition of gradient echo scans were also performed to acquire highresolution 3D anatomic images (TR, $2300 \mathrm{~ms}$; TE, $2.98 \mathrm{~ms}$; TI, 900 ms; flip angle, $9^{\circ}$; resolution, $\left.1 \times 1 \times 1 \mathrm{~mm}^{3}\right)$. DTI data with 3 b-values $\left(0,500\right.$, and $\left.1000 \mathrm{~s} / \mathrm{mm}^{2}\right)$ and 30 standard directions (spatial resolution of $1.32 \times 1.32 \times 4 \mathrm{~mm}^{3}$ ) with similar coverage space as the fMRI and parallel acquisition direction were also obtained. Conventional imaging, including T2-weighted fast spin-echo and fluid-attenuated inversion recovery images (TR, $9200 \mathrm{~ms}$; TE, $82 \mathrm{~ms}$; TI, $2500 \mathrm{~ms}$; flip angle, $150^{\circ}$; resolution, $0.86 \times 0.86 \times 3 \mathrm{~mm}^{3}$ ), was also obtained for lesion load quantification. Gadolinium-enhanced T1-weighted imaging (TR, 354 ms; TE, $2.73 \mathrm{~ms}$; flip angle, $56^{\circ}$; resolution, $0.3 \times 0.3 \times 3 \mathrm{~mm}^{3}$ ) was performed to detect any enhancing active plaques in patients with MS.

\section{Image Processing and Data Analysis}

Anatomic 3D-magnetization-prepared rapid acquisition of gradient echo and functional imaging data were preprocessed by both FSL (http://www.fmrib.ox.ac.uk/fsl) and AFNI (http://afni. nimh.nih.gov/afni/) (scripts containing the processing procedures are available via the 1000 Functional Connectome Project: http://fcon_1000.projects.nitrc.org). Preprocessing steps included spatial smoothing with a full width at half maximum of 6 $\mathrm{mm}$, band pass temporal filtering of $0.005-0.1 \mathrm{~Hz}$ to remove physiologic noise contamination, ${ }^{15}$ removal of nuisance signals with regression model (motion parameters, the global signal, and signals derived from CSF and white matter), and transformation to Montreal Neurological Institute 152 standard space $(2 \times 2 \times 2$ $\mathrm{mm}^{3}$ ) with the nonlinear optimization warping algorithm called FMRIB's Nonlinear Image Registration Tool (FNIRT; http://fsl.fmrib.ox.ac.uk/fsl/fslwiki/FNIRT). In our study, we have evaluated physiologic noise by using temporal independent component analysis in each group with a standard FSL-MELODIC algorithm (http://www.fmrib.ox.ac.uk/fsl/melodic/index.html). We found that the physiologic noise pattern was typical ${ }^{16}$ (eg, respiratory noise with peak power spectrum at $0.2 \mathrm{~Hz}$ ) and similar 
in the 2 groups; therefore, the bandpass filtering $(0.005-0.1 \mathrm{~Hz})$ applied in this study can effectively remove these noises. In addition, we used an unbiased physiologic noise template ${ }^{17}$ and dual regression model to map the noise pattern in each group and found no significant spatial distribution differences between these 2 groups. This finding suggests that the possible altered autonomic functions in MS do not contribute to the physiologic noise in this patient group and are unlikely to influence the homotopic analysis in this study.

For correction of motion artifacts, a head movement-constraint headphone and cushion were used to prevent movement during the scan, and all participants were well-instructed to keep still during the RS-fMRI scan. Using AFNI motion correction algorithms, we performed more realignment steps with 3 angular rotation (roll, pitch, and yaw in the units of degrees) and 3 directional displacements (unit: millimeters) to further minimize the motion artifacts. After these correction steps, no statistical differences $(P=.53)$ of average frame displacement (sum of all motion parameters) ${ }^{18}$ were observed between the MS group $(0.163 \pm 0.064 \mathrm{~mm})$ and the control group $(0.149 \pm$ $0.083 \mathrm{~mm}$ ).

VMHC, the Pearson correlation between the preprocessed time-series of each pair of symmetric interhemispheric voxels, was computed according to the procedures outlined in our previous study. ${ }^{2}$ The resultant values were $z$-transformed (by use of the Fisher r-to-z transform) and were used for subsequent grouplevel analysis. We used a 2-sample Student $t$ test to compare VMHC between the patient group and the healthy control group. The participant's age and sex were used as covariants for comparison to derive the results. Multiple comparison corrections at the cluster level were performed to the whole brain based on Gaussian random field theory by the FSL easythresh command (minimum $Z>2.3$; cluster significance, $P<.05$, corrected).

The global VMHC score for each participant was obtained by the average of the $\mathrm{VMHC}$ z-images of each participant within the $25 \%$ gray matter mask from the FSL template for further quantification. Regional VMHC values were obtained based on the $50 \%$ and 112 total parcellation masks of the left and right hemispheric regions, ${ }^{1}$ with in-house developed scripts according to FSL and Matlab software (MathWorks, Natick, Massachusetts). These brain regions are further classified into 3 functional hierarchic subdivisions, ${ }^{19}$ namely primary sensory-motor, unimodal association, and heteromodal association areas. Then average values of each hierarchy can be quantified as described by Stark et $\mathrm{al}^{1}$ to compare between 2 groups. The primary motor and sensory cortices are engaged directly with the environment including the postcentral gyrus (somatosensory), intracalcarine cortex and occipital pole (visual), Heschl gyrus (auditory), and precentral gyrus (motor). Unimodal association areas are those regions adjacent to the primary sensory-motor cortices involved in integration of information from predominantly 1 sensory or motor technique (eg, supramarginal gyrus, occipital fusiform gyrus). The heteromodal association areas, located primarily in the prefrontal and temporoparietal cortices, integrate information from multiple sensory and motor modalities.

For CC volumetry, a midsagittal CC skeleton was delineated in the middle sagittal section after reorientation of $3 \mathrm{D}$ magnetiza- tion-prepared rapid acquisition of gradient echo images to the anterior/posterior commissure coordinate system by use of MEDx software (www.medicalnumerics.com), and the CC area was derived. For CC FA quantification, the FSL tract-based spatial statistics toolbox ${ }^{20}$ steps 1-2 was used for registration of all participants' FA into the FSL template. The FA data were transformed to the FSL Montreal Neurological Institute (1-mm isotropic resolution) common space by use of the nonlinear registration tool FNIRT based on a b-spline representation of the registration warp field. Whole CC was obtained from the FSL Jülich atlas (http://fsl.fmrib.ox.ac.uk/fsl/fsl4.0/ fslview/atlas-descriptions.html\#jul). Segmentation of the CC into 5 segments was done in MEDx in the template space according to the calculated proportional distance ${ }^{21}$ in the sagittal view and was translated to the axial view. Average FA of the CC was performed to quantify structural properties of the $\mathrm{CC}$ and to correlate with global or regional mean VMHC values (Pearson correlation). In addition, Spearman rank correlations were performed between Expanded Disability Status Scale and FA or global VMHC measurements.

Using high-resolution 30-direction DTI data, we performed seed-based fiber tractography by using Diffusion Toolkit (http:// www.nitrc.org/projects/trackvis), with an advanced tensorline propagation algorithm and standard threshold setting (eg, 35degree angle threshold for tensor propagations). The tensorline algorithm can incorporate both the anisotropic classification of the local tensor and the nearby fiber orientation information into the tracking algorithm; therefore, it is more robust and reproducible in regions that are either noisy or have crossing fibers. ${ }^{22,23}$ To evaluate the relationship between the interhemispheric and intrahemispheric structural and functional connectivity of cortical motor regions, a $4.5-\mathrm{mm}$ radius sphere was placed as a seed in the posterior motor triangle area to delineate both transcallosal (interhemispheric) and nontranscallosal CST (intrahemispheric) motor fibers. Homotopic values were obtained from the terminals of either the CC-connected fibers (mainly in the cortical motor regions) or CST projections (mainly in the premotor regions) to compare the VMHC differences of the homotopic vs heterotopic regions.

FireVoxel software (https://files.nyu.edu/hr18/public) was used for MS lesion load quantification, after intensity uniformity correction of the fluid-attenuated inversion recovery images. The process was started by automatic detection of white matter intensity in a periventricular normal-appearing white matter "seed" region on 3 continuous sections. After the calculation of the seedbased "normal" white matter intensity, appropriate thresholds $(\geq$ mean $+2.5 \mathrm{SD})$ were selected and applied to all voxels, and an abnormal tissue mask was constructed per section. This process involved 3 steps: 1) morphologic erosion, 2) recursive region growth-retaining pixels connected to the "seed," and 3) morphologic inflation to reverse the effect of erosion. Lesion volumes were then obtained from each patient after confirmation and correction by an experienced radiologist. In patients with MS, we performed correlation analysis between FA of the entire CC or global VMHC and lesion volume by using the Pearson correlation. 
FIG 1. Whole-brain voxelwise homotopic RSFC pattern by use of multiple linear regression models in control $(A)$ and MS $(B)$ groups. Homotopic RSFC was computed within 1 hemisphere (left side) for each pair of homotopic voxels and corrected by the Gaussian random field theory (minimum $Z>2.3$; cluster level, $P<.05$, corrected). The final statistical maps are visualized as 6 hemispheric surfaces (cortical regions) with 6-mm full width at half maximum and multiple axial images (subcortical regions). Compared with control participants, patients demonstrated decreased VMHC in several cortical regions (long arrows) including the frontal, temporal, and occipital lobes and increased VMHC mainly in the subcortical regions (short arrows).

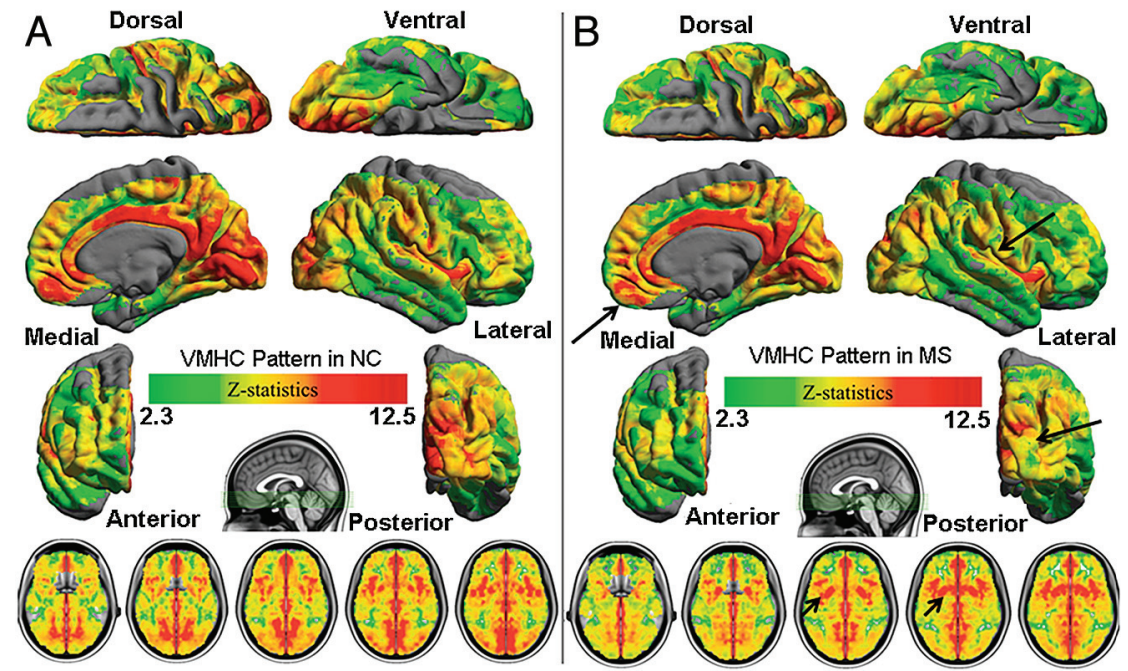

and a significant decrease of VMHC was only found in the primary cortices in the MS group compared with the control group (Fig 3B).

We also observed the reduction of the midsagittal area of the $\mathrm{CC}(P=.026)$ (Fig $4 A)$ and FA of the entire CC $(P=.0018)$ (Fig $4 B$ ) in patients compared with control particpants. The global VMHC correlates moderately with the average FA of the entire CC for all participants in both groups $(r=0.3 ; P=.03$ ) (Fig $5 A)$, however, without statistical significance in each separate group. In addition, we found decreased FA in each of the 5 CC segments $(P \leq .02)$, indicating a change in whole-brain CC integrity in patients compared with control participants. In the MS group, a significant positive correlation was also found between average VMHC in the occipital regions and average FA values of CC segment 5 corresponding to occipital projections $(r=$ $0.43 ; P=.037)($ Fig $5 B)$. There was a sig-

\section{RESULTS}

In healthy control participants, several primary cortical regions including the visual, motor, and somatosensory cortices showed the strongest VMHC, whereas the prefrontal and temporoparietal-associated cortices and the paracingulate areas demonstrated relatively weaker VMHC. The dorsolateral and ventrolateral portions in particular, which are known for characteristic lateralization of function related to language, attention, and cognitive control (Fig $1 A$ ), exhibited this weakness.

In patients with MS, no enhancing lesion was observed on gadolinium-enhanced T1-weighted imaging. These patients exhibited an altered global VMHC pattern compared with control participants. Specifically, there was decreased VMHC within many higher-order cognitive regions, including the frontal, temporal, and occipital lobes (Fig $1 B$ ), in patients compared with control participants (corrected $P<.05$ ) (Fig 2). There was also increased VMHC in the anterior insular, thalamus, striatum, cerebellum, and inferior temporal regions in patients compared with control participants (Fig 2), and these regions are mainly associated with sensory processing and motor control. A detailed comparison of VMHC in different regions between the 2 groups is shown in Tables 1 and 2. Overall, there was a decreased global VMHC in the MS group compared with that in the control group $(P=.04)$ (Fig 3A), indicating a deficit of whole-brain interhemispheric synchronization in the patients. Regional interhemispheric VMHC analysis found the highest values in the visual cortex followed by the somatosensory, auditory, and motor areas in both the MS group and the control group. There was also significant reduction of VMHC in the visual, somatosensory, and motor (subregions) cortices $(P=.04)$ in the patient group. In addition, VMHC showed significantly higher homotopic connectivity in the primary cortices compared with unimodal and heteromodal regions in both the MS group and the control group, nificant negative correlation between patients' Expanded Disability Status Scale score and the average FA of the CC $(r=-0.61$; $P=.013$ ) (Fig $5 C$ ). The average FA of the CC also significantly correlated negatively with patients' lesion load $(r=-0.92 ; P<$
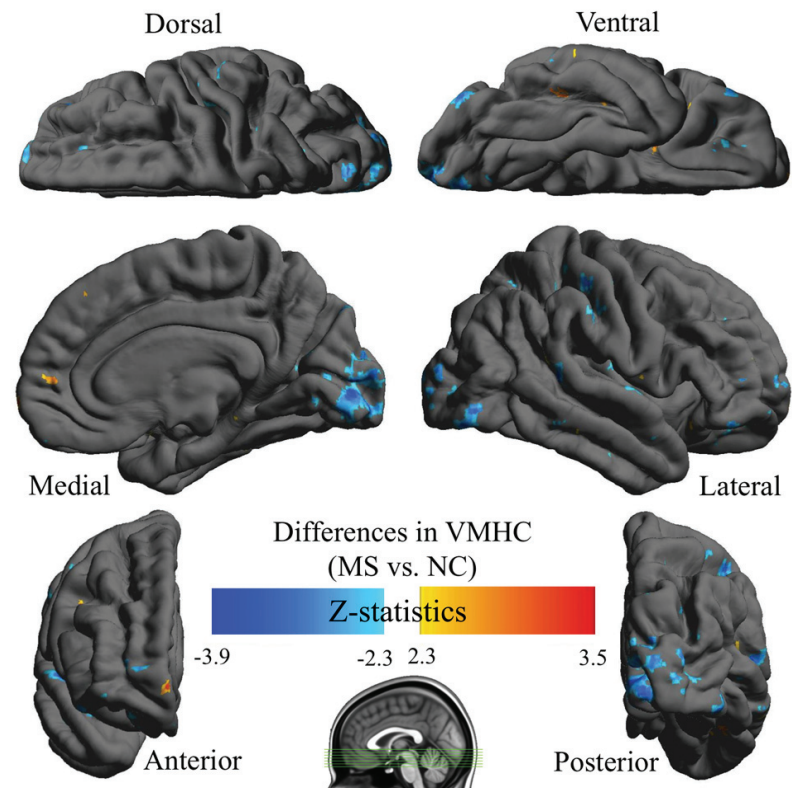

Differences in VMHC (MS vs. NC)

Z-statistics
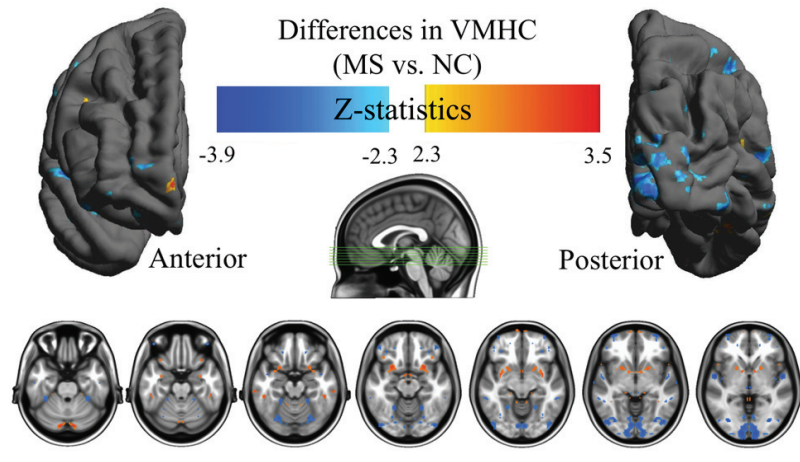

FIG 2. Comparison results of whole-brain voxelwise VMHC maps between patients with MS and healthy control participants $(N C)$ showed regions with significantly decreased (blue) and increased VMHC (red and yellow) in patients compared with control participants corrected with Gaussian random field theory (minimum $Z>2.3$; cluster level; $P<.05$, corrected).

AJNR Am J Neuroradiol 34:1180-87 Jun 2013 www.ajnr.org 1183 
Table 1: Brain regions showing decreased VMHC in patients with MS vs control participants

\begin{tabular}{|c|c|c|c|c|}
\hline Brain Region & BA & $\begin{array}{c}X, Y, Z, M N I \\
(\mathrm{~mm})\end{array}$ & $\begin{array}{c}\text { Cluster } \\
\text { Size }\end{array}$ & $\begin{array}{l}\text { Maximal Z } \\
\text { Score }\end{array}$ \\
\hline Visual (calcarine) & 17 & $-10,-94,-2$ & 905 & 3.83 \\
\hline Primary somatosensory & 1 & $-50,-16, \quad 46$ & 286 & 3.8 \\
\hline Lateral occipital & 19 & $-34,-74,-4$ & 163 & 3.58 \\
\hline Middle temporal & 22 & $-66,-46, \quad 8$ & 129 & 4.07 \\
\hline Frontal pole & 11 & $-36,64,-6$ & 126 & 3.52 \\
\hline Superior temporal & 48 & $-54,-4,-2$ & 93 & 3.88 \\
\hline Inferior occipital & 18 & $-24,-70,-16$ & 65 & 3.41 \\
\hline Middle occipital & 37 & $-38,-42,-28$ & 64 & 3.06 \\
\hline Fusiform & 37 & $-22,-54,-14$ & 59 & 3.35 \\
\hline Cuneus & 19 & $-12,-82, \quad 34$ & 57 & 3.68 \\
\hline
\end{tabular}

Note:-Only the right side is listed because of symmetric computation between the hemispheres, $P$-values were obtained with cluster-level multiple comparison correction (corrected $P<.05$; cluster size $>20$ ); BA indicates Brodmann area; MNI, Montreal Neurological Institute.

Table 2: Brain regions showing increased VMHC in patients with MS vs control participants

\begin{tabular}{lcccc}
\hline \multicolumn{1}{c}{ Brain Region } & BA & $\begin{array}{c}\boldsymbol{X}, \boldsymbol{Y , Z , M N I} \mathbf{M N} \\
(\mathbf{m m})\end{array}$ & $\begin{array}{c}\text { Cluster } \\
\text { Size }\end{array}$ & $\begin{array}{c}\text { Maximal Z } \\
\text { Score }\end{array}$ \\
\hline Orbital frontal/insular & 48 & $-26,-12,-14$ & 160 & 3.45 \\
Thalamus & - & $-2,-24, \quad 12$ & 40 & 3.16 \\
Cerebellum & - & $-10,-82,-26$ & 40 & 3.44 \\
Pallidum & - & $-10,4,-4$ & 23 & 3.19 \\
Inferior temporal & 20 & $-48,-34,-20$ & 22 & 3.71 \\
\hline
\end{tabular}

Note:-Only the right side is listed because of symmetric computation between the hemispheres. $P$-values were obtained with cluster-level multiple comparison correction (corrected, $P<.05$; cluster size $>20$ ); BA indicates Brodmann area; MNI, Montreal Neurological Institute.

.0001) (Fig 5D). No significant correlation was found between global homotopic VMHC changes and Expanded Disability Status Scale or lesion load.

The homotopic degree of VMHC of the motor cortex connected with either transcallosal CC fibers (ie, interhemispheric) or nontranscallosal lateral CST (intrahemispheric), according to fiber tractography seeding with a spheric region of interest near the CC body, was evaluated. Higher VMHC was observed in the cortical motor regions with direct transcallosal vs nontranscallosal fiber connection ( 0.68 vs 0.29 , respectively) (Fig $6 A, B)$. The statistical comparison of transcallosal VMHC and nontranscallosal VMHC showed a significant difference between the 2 terminal regions connected with transcallosal and nontranscallosal fibers in each group $(P<.001)$, and a lower value was found in nontranscallosal regions. In addition, there was significantly reduced VMHC in the transcallosal motor regions $(P=.03)$, but not in the nontranscallosal (ie, CST) regions $(P=.09)$, in patients compared with control participants (Fig 6C).

\section{DISCUSSION}

The results of our study showed significant changes of functional interhemispheric correlation measured with VMHC in patients with MS, which may have important clinical implications such as disturbances of visual, sensory, and motor coordination as well as various cognitive deficits (eg, information processing speed, attention, coherent volition, etc), in these patients. The regions having the largest reductions of $\mathrm{VMHC}$ were observed in the middle and lateral parts of the occipital lobe, which consist mainly of the primary visual cortex, but also include the primary somatosensory cortex and areas in the temporal and frontal lobes. Dysfunction of these regions is responsible for the sensory, movement, or memory deficits in patients with MS. In addition, our analysis of the relationship between homotopic changes and CC microstructural FA changes suggests that, as callosal degeneration increases, the functional homotopy decreases, which may be indicative of inefficient callosal transmission in patients. Our data also support the concept that increased functional coupling between the hemispheres can emerge in the presence of callosal pathologic features with disruption of anatomic connectivity. ${ }^{24}$

Despite the potential importance of assessing the deficits of interhemispheric coordination in MS, no detailed study of imaging global functional homotopy has been performed. The VMHC we used quantifies the global RSFC between each voxel in 1 hemisphere and its mirrored counterpart in the other. The pattern of VMHC in the healthy control participants from our study is consistent with recently published studies showing highest symmetric synchronization in the occipital and somatosensory cortices and in many midline brain structures including the striatum, cingulate, and thalamus. ${ }^{2,4}$ In MS, by using a voxel-based RSFC approach to analyze synchrony in spontaneous activity between the geometrically corresponding interhemispheric regions, we found significantly reduced VMHC in areas of the occipital, frontal, and temporal lobes in patients compared with control participants. Our functional hierarchic analysis showed that homotopic deficits mainly involved the primary visual and somatosensory cortices in patients with MS, ${ }^{1}$ which are consistent with these typical clinical manifestations that are often seen in this disease. However, the unimodal and heteromodal regions, which usually have a relatively lower degree of interhemispheric correlations as found in our current and previous study, ${ }^{1}$ showed no
FIG 3. Comparison results of global $(A)$ and regional $(B) \mathrm{VMHC}$ differences between patients with MS and healthy control participants. There was significantly reduced global $\mathrm{VMHC}$ in patients compared with control participants $(P=.04)(A)$. Regional analysis showed significantly higher interhemispheric correlation in the primary cortical regions (ie, visual, somatosensory, motor, and auditory cortices) compared with unimodal and heteromodal regions $(B)$ in both groups. Significantly lower VMHC was found only in the primary cortex in patients compared with control participants $(P<.05)$. Error bars denote standard error in each group. 

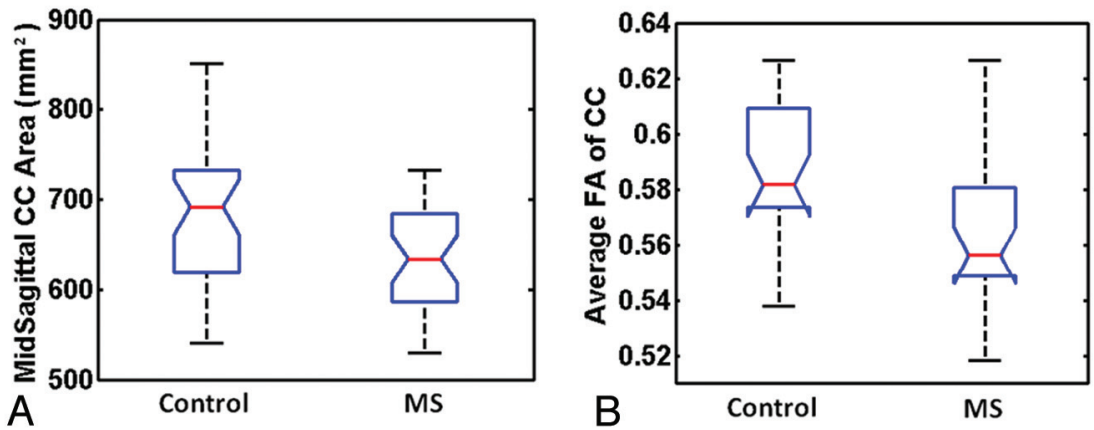

FIG 4. Structural differences measured with midsagittal $C C$ area $(A)$ and the FA of the entire $C C$ $(B)$ between patients with MS and control participants showed a significantly reduced midsagittal CC area $(P=.026)$ and FA $(P=.0018)$ in patients compared with control participants. The boxes have lines at the lower quartile (horizontal blue lines), median (horizontal red lines), and upper quartile values. The whiskers are lines extending from each end of the boxes to show the extent of the rest of the data. The "notch" marks the $95 \%$ confidence interval for the medians, which can be used to differentiate 2 groups. Namely 2 medians will differ significantly with $P<$ .05 if the 2 notch intervals do not overlap.
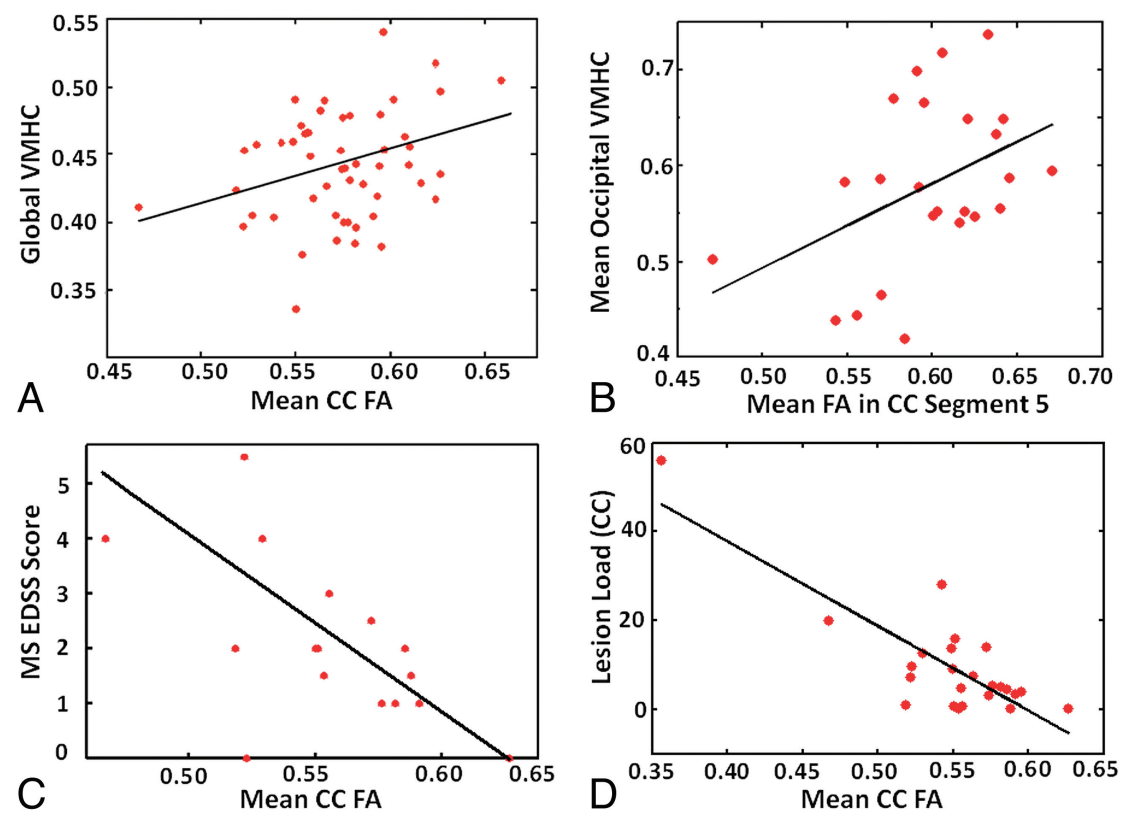

FIG 5. Mean FA of the entire $C C$ and global VMHC in all participants $(A)$ showed significant positive correlation ( $r=0.3 ; P=.03$ ). Mean FA of CC segment 5 (occipital projections) and mean $\mathrm{VMHC}$ in the occipital regions showed significant positive correlation $(r=0.43 ; P=.037)$ in patients with MS $(B)$. There was a significant negative correlation between the Expanded Disability Status Scale score and the mean FA of CC $(r=-0.61 ; P=.013)$ in the patients $(C)$. The mean FA of CC also significantly correlates with the lesion load $(r=-0.92 ; P<.0001)(D)$. mechanism could also lead to greater activation in these regions in MS. ${ }^{25}$

Although our global analysis showed that structural connectivity measured by FA of the entire CC correlates with global interhemispheric homotopy in all participants, we did not find the significant correlation in the patients alone. This is consistent with the findings of Lowe et al, ${ }^{13}$ who reported increased FA with increased functional connectivity in all participants but not in the patient group. This was likely because the decreased functional connectivity can be accompanied by compensatory increased connectivity, as found in this study, and our global measure may not be specific enough to exhibit a high correlation with a limited number of patients. To date, a limited number of studies have tried to correlate interhemispheric structural and functional connectivity, and it is still unclear to what degree they may be related in healthy and diseased conditions. Johnston et $\mathrm{al}^{26}$ presented a striking loss of interhemispheric functional connectivity with preserved intrahemispheric correlations in a 6-yearold child after complete CC section for the treatment of intractable epilepsy. However, several studies ${ }^{24,27}$ showed preserved interhemispheric functional connectivity in cases of agenesis of the CC. The different results reported from the above studies may thus arise from several factors: 1) compensatory functional reorganization $^{28}$ ensues in both subcortical and cortical synchronization in the absence of a CC, 2) not all regions require direct structural connectivity for normal functional coherence, and 3) structural connectivity only partially shapes functional connectivity for functional compensation in a slow and evolutionary fashion.

significant difference of VMHC between patients and control participants.

Our current study also demonstrated increased VMHC in the subcortical areas, including the thalamus, insula, pallidum, and cerebellum, in patients compared with control participants, despite the reduction of overall global VMHC. Many of these increased regions are connected via the subcortical connections (eg, thalamus projections) or other interhemispheric connections, (eg, anterior/posterior commissure for inferior temporal connections or CSTs). These noncallosal interhemispheric homotopic increases are assumed to compensate for the decreased interhemispheric coordination when transcallosal function is impaired in patients with MS. The disruption of the transcallosal inhibitory
In our study, we chose 2 neighboring interhemispheric primary motor regions to further study homotopic vs heterotopic correlations and to evaluate a specific functional pathway that is connected either with transcallosal (homotopic) or nontranscallosal (heterotopic or intrahemispheric) fiber tracts on the basis of DTI tractography. We found that VMHC is significantly higher in homotopic regions (with transcallosal connection) vs heterotopic regions (without transcallosal connection), indicating that baseline (resting-state) bilateral cortical coactivation is higher in homotopic regions. However, such structural-function relationships may have varying levels of interdependence across regions and may also depend on different functional pathways. In a study of language networks, Morgan et $\mathrm{al}^{29}$ showed no correlations be- 

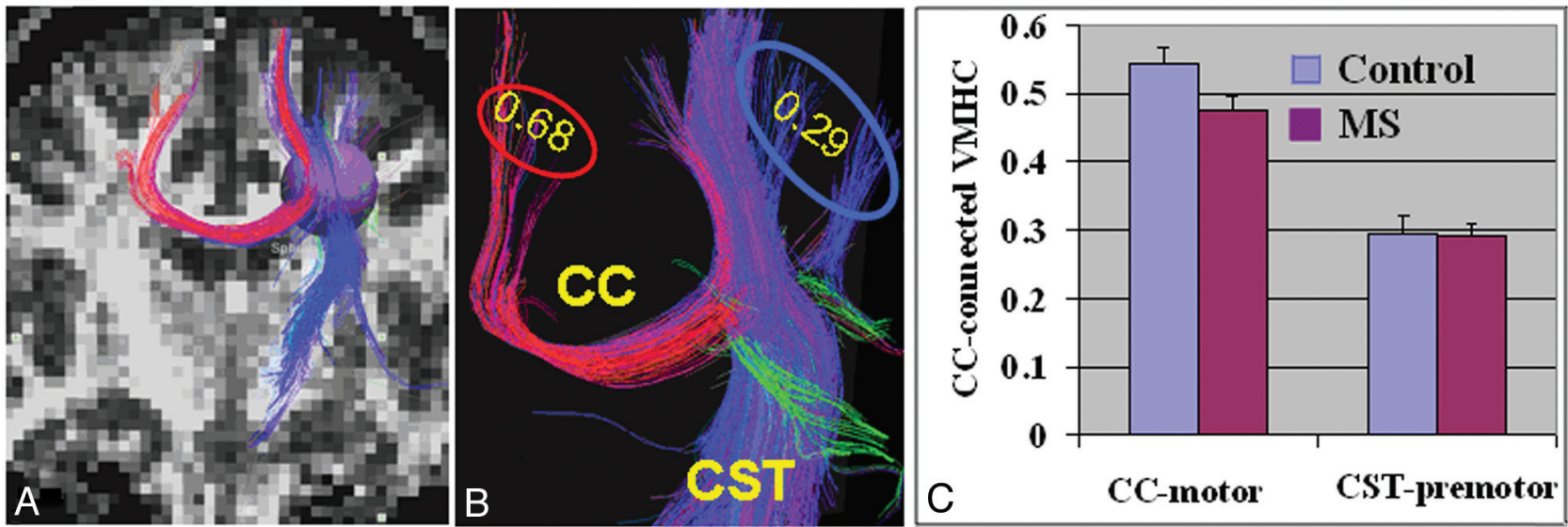

FIG 6. Interhemispheric and intrahemispheric structural and functional connectivity of cortical motor regions. A, On the basis of whole-brain fiber tractography from FA images, a sphere with a radius of $4.5 \mathrm{~mm}$ was placed in the posterior motor triangle area as a seed to select both transcallosal (interhemispheric fibers indicated in red) and nontranscallosal CST (intrahemispheric fibers indicated in blue) motor fibers to define the connecting terminal cortices for VMHC evaluation. $B$, A representative example of different VMHC values in 2 separate terminal regions showed higher VMHC (0.68) in motor regions connected with transcallosal fibers compared with premotor regions $(0.29)$ projected by nontranscallosal CST fibers. C, Group comparison of transcallosal VMHC and nontranscallosal VMHCs showed much higher value in transcallosal regions. There was significantly reduced VMHC in transcallosal motor regions in patients compared with control participants $(P=.03)$ but not in nontranscallosal CST regions $(P=.09)$.

tween FA and functional connectivity along the pathways connecting either Broca area and supplementary area or Broca area and Wernicke area. Our results showed the most prominent decrease of VMHC in the primary visual cortex of patients with MS, and the reduced $\mathrm{VMHC}$ in these regions correlated significantly with the FA of CC segment 5 for occipital projections. This finding merges with the clinical symptom of a relatively prominent visual functional deficit previously or recently occurring in these patients, suggesting that decreased VMHC is possibly attributed to a callosal degenerative pathologic state with axonal attenuation. ${ }^{30}$ Our results also showed a higher degree of VMHC decrease in patients with lower callosal FA; such decreased VMHC is more prominent in homotopic vs heterotopic regions, which is consistent with our previous findings of preferential involvement of the $\mathrm{CC}$ in $\mathrm{MS}^{6}{ }^{6}$ Similar to our findings, Lowe et $\mathrm{al}^{13}$ reported that functional connectivity by RS-fMRI is reduced between the bilateral primary sensorimotor regions in MS but did not reach a significant difference between 11 patients with MS and 10 control participants. In line with the results in this study, we also demonstrated a correlation between damage of the transcallosal motor pathway measured with DTI and its terminal bilateral sensorimotor functional connectivity in MS. In contrast to these results, Rocca et al ${ }^{14}$ reported increased interhemispheric functional connectivity of the sensorimotor network with CC damage in patients with MS. This discrepancy may be because different techniques were used in these studies, with our study using VMHC with perfect symmetry.

\section{CONCLUSIONS}

Our data provide preliminary evidence of the potential usefulness of VMHC analyses for the detection of abnormalities of interhemispheric coordination in MS. We demonstrated that the whole-brain homotopic RS-fMRI pattern was altered in patients with MS, which was partially associated with underlying structural degenerative changes of the CC measured with FA. Such observations can increase our understanding of interhemispheric structural and functional deficits in MS.

\section{ACKNOWLEDGMENTS}

We thank Lin Tang for her assistance with this manuscript.

Disclosures: Yongxia Zhou-RELATED: Grant: National Institutes of Health (NIH Grant \#: R01NS029029).* Robert I. Grossman—RELATED: Grant: NIH. * Yulin GeRELATED: Grant: NIH, ${ }^{*}$ Comments: This study was supported by Award no. R01NS029029 from the NIH. (*Money paid to institution.)

\section{REFERENCES}

1. Stark DE, Margulies DS, Shehzad ZE, et al. Regional variation in interhemispheric coordination of intrinsic hemodynamic fluctuations. J Neurosci 2008;28:13754-64

2. Zuo XN, Kelly C, Di Martino A, et al. Growing together and growing apart: regional and sex differences in the lifespan developmental trajectories of functional homotopy. J Neurosci 2010;30:15034-43

3. Fair DA, Dosenbach NU, Church JA, et al. Development of distinct control networks through segregation and integration. Proc Natl Acad Sci U S A 2007;104:13507-12

4. Kelly C, Zuo XN, Gotimer K, et al. Reduced interhemispheric resting state functional connectivity in cocaine addiction. Biol Psychiatry 2011;69:684-92

5. Brown LN, Zhang Y, Mitchell JR, et al. Corpus callosum volume and interhemispheric transfer in multiple sclerosis. Can J Neurol Sci 2010;37:615-19

6. Ge Y, Law M, Johnson G, et al. Preferential occult injury of corpus callosum in multiple sclerosis measured by diffusion tensor imaging. J Magn Reson Imaging 2004;20:1-7

7. Sigal T, Shmuel M, Mark D, et al. Diffusion tensor imaging of corpus callosum integrity in multiple sclerosis: correlation with disease variables. J Neuroimaging 2012;22:33-37

8. Aboitiz F, Montiel J. One hundred million years of interhemispheric communication: the history of the corpus callosum. Braz J Med Biol Res 2003;36:409-20

9. Kennerley SW, Diedrichsen J, Hazeltine E, et al. Callosotomy patients exhibit temporal uncoupling during continuous bimanual movements. Nat Neurosci 2002;5:376-81

10. Desjardins S, Braun CM. Homotopy and heterotopy and the bilat- 
eral field advantage in the Dimond paradigm. Acta Psychol (Amst) 2006;121:125-36

11. Ge Y, Law M, Grossman RI. Applications of diffusion tensor MR imaging in multiple sclerosis. Ann N Y Acad Sci 2005;1064:202-19

12. Chao YP, Cho KH, Yeh CH, et al. Probabilistic topography of human corpus callosum using cytoarchitectural parcellation and high angular resolution diffusion imaging tractography. Hum Brain Mapp 2009;30:3172-87

13. Lowe MJ, Beall EB, Sakaie KE, et al. Resting state sensorimotor functional connectivity in multiple sclerosis inversely correlates with transcallosal motor pathway transverse diffusivity. Hum Brain Mapp 2008;29:818-27

14. Rocca MA, Absinta M, Moiola L, et al. Functional and structural connectivity of the motor network in pediatric and adult-onset relapsing-remitting multiple sclerosis. Radiology 2010;254:541-50

15. Beall EB, Lowe MJ. The non-separability of physiologic noise in functional connectivity MRI with spatial ICA at 3T. JNeurosci Methods 2010;191:263-76

16. Beall EB. Adaptive cyclic physiologic noise modeling and correction in functional MRI. J Neurosci Methods 2010;187:216-28

17. Zuo XN, Kelly C, Adelstein JS, et al. Reliable intrinsic connectivity networks: test-retest evaluation using ICA and dual regression approach. Neuroimage 2010;49:2163-77

18. Power JD, Barnes KA, Snyder AZ, et al. Spurious but systematic correlations in functional connectivity MRI networks arise from subject motion. Neuroimage 2012;59:2142-54

19. Mesulam M. Brain, mind, and the evolution of connectivity. Brain Cogn 2000;42:4-6

20. Smith SM, Jenkinson M, Johansen-Berg H, et al. Tract-based spatial statistics: voxelwise analysis of multi-subject diffusion data. Neuroimage 2006;31:1487-505

21. Rosas HD, Lee SY, Bender AC, et al. Altered white matter microstructure in the corpus callosum in Huntington's disease: implications for cortical "disconnection". Neuroimage 2010;49:2995-3004

22. Lazar M, Weinstein DM, Tsuruda JS, et al. White matter tractography using diffusion tensor deflection. Hum Brain Mapp 2003;18:306-21

23. Mori S, van Zijl PC. Fiber tracking: principles and strategies-a technical review. NMR Biomed 2002;15:468-80

24. Tyszka JM, Kennedy DP, Adolphs R, et al. Intact bilateral restingstate networks in the absence of the corpus callosum. J Neurosci 2011;31:15154-62

25. Lenzi D, Conte A, Mainero C, et al. Effect of corpus callosum damage on ipsilateral motor activation in patients with multiple sclerosis: a functional and anatomical study. Hum Brain Mapp 2007;28:636-44

26. Johnston JM, Vaishnavi SN, Smyth MD, et al. Loss of resting interhemispheric functional connectivity after complete section of the corpus callosum. J Neurosci 2008;28:6453-58

27. Khanna PC, Poliakov AV, Ishak GE, et al. Preserved interhemispheric functional connectivity in a case of corpus callosum agenesis. Neuroradiology 2012;54:177-79

28. Cifelli A, Matthews PM. Cerebral plasticity in multiple sclerosis: insights from fMRI. Mult Scler 2002;8:193-99

29. Morgan VL, Mishra A, Newton AT, et al. Integrating functional and diffusion magnetic resonance imaging for analysis of structurefunction relationship in the human language network. PLoS One 2009;4:e6660

30. Evangelou N, Konz D, Esiri MM, et al. Regional axonal loss in the corpus callosum correlates with cerebral white matter lesion volume and distribution in multiple sclerosis. Brain 2000;123:1845-49 